\title{
On the Efficiency of the Proportional Allocation Mechanism for Divisible Resources
}

\author{
George Christodoulou ${ }^{1} \cdot$ Alkmini Sgouritsa $^{1}$ (D) . \\ Bo Tang ${ }^{1}$
}

(C) The Author(s) 2016. This article is published with open access at Springerlink.com

\begin{abstract}
We study the efficiency of the proportional allocation mechanism that is widely used to allocate divisible resources. Each agent submits a bid for each divisible resource and receives a fraction proportional to her bids. We quantify the inefficiency of Nash equilibria by studying the Price of Anarchy (PoA) of the induced game under complete and incomplete information. When agents' valuations are concave, we show that the Bayesian Nash equilibria can be arbitrarily inefficient, in contrast to the well-known $4 / 3$ bound for pure equilibria Johari and Tsitsiklis (Math. Oper. Res. 29(3), 407-435 2004). Next, we upper bound the PoA over Bayesian equilibria by 2 when agents' valuations are subadditive, generalizing and strengthening previous bounds on lattice submodular valuations. Furthermore, we show that this bound is tight and cannot be improved by any simple or scale-free mechanism. Then we switch to settings with budget constraints, and we show an improved upper bound on the PoA over coarse-correlated equilibria. Finally, we prove that the PoA is exactly 2 for pure equilibria in the polyhedral environment.
\end{abstract}

The first author was supported by EP/M008118/1, EP/K01000X/1 and LT140046.

Alkmini Sgouritsa

a.sgouritsa@liverpool.ac.uk

George Christodoulou

gchristo@liverpool.ac.uk

Bo Tang

bo.tang@liverpool.ac.uk

1 University of Liverpool, Liverpool, UK 


\section{Introduction}

Allocating network resources, like bandwidth, among agents is a canonical problem in the network optimization literature. A traditional model for this problem was proposed by Kelly [18], where allocating these infinitely divisible resources is treated as a market with prices. More precisely, agents in the system submit bids on resources to express their willingness to pay. After soliciting the bids, the system manager prices each resource and then agents buy portions of resources by paying a proportional amount of the prices. The users act as price takers, trying to maximize their utility, i.e. the difference between their valuations and payments, and they do not anticipate the effect of their actions on the prices. Kelly [18] showed that, under certain assumptions, the aggregate utility of the users is maximized when the players receive portions of the resources that are proportional to their bids. In the case of a single resource, each user receives a fraction of the resource equal to the ratio of their bid over the sum of all bids; additionally, they should pay an amount equal to their own bid. This is known as the proportional allocation mechanism or Kelly's mechanism in the literature. It is widely used in network pricing and has been implemented for allocating computing resources in several distributed systems [8].

Johari and Tsitsiklis [16] relaxed the assumption that the users act as price takers and instead they can anticipate the effects of their actions on the prices of the resources. They observed that this strategic bidding in the proportional allocation mechanism leads to inefficient allocations that do not maximize social welfare. On the other hand, they showed that this efficiency loss is bounded when agents' valuations are concave. More specifically, they proved that the proportional allocation mechanism admits a unique pure Nash equilibrium with Price of Anarchy (PoA) [19] at most $4 / 3$. The inefficiency of equilibria has been also observed in other network games such as routing games [19], routing traffic games [25], congestion games [24] and network design games $[1,4]$.

An essential assumption used by Johari and Tsitsiklis [16] is that agents have complete information of each other's valuations. However, in many realistic scenarios, the agents are only partially informed. A standard way to model incomplete information is by using the Bayesian framework, where the agents' valuations are drawn independently from some publicly known distribution that, in a sense, represents the agents' beliefs. A natural question is whether the efficiency loss is still bounded in the Bayesian setting. We give a negative answer to this question by showing that the PoA over Bayesian equilibria is at least $\sqrt{m} / 2$, where $m$ is the number of resources. This result complements the recent study by Caragiannis and Voudouris [3], where the PoA of single-resource proportional allocation games is shown to be at most 2 in the Bayesian setting.

Non-concave valuation functions were studied by Syrgkanis and Tardos [26] for both complete and incomplete information games. They showed that, when agents' valuations are lattice-submodular, the PoA for coarse correlated and Bayesian Nash equilibria is at most 3.73 by applying their general smoothness framework. In this paper, we study subadditive valuations [12] that is a superclass of lattice submodular functions. We prove that the PoA over Bayesian Nash equilibria is at most 2. Moreover, we show optimality of the proportional allocation mechanism by showing that 
this bound is tight and cannot be improved by any simple mechanism, as defined in the recent framework of Roughgarden [23], ${ }^{1}$ or any scale-free mechanism. ${ }^{2}$

Next, we switch to the setting where agents are constrained by budgets that represent the maximum payment they can afford. We use as a benchmark the effective welfare $[3,10,26]$, capping the contribution of each player to the welfare by their budget (in [10], they called it liquid welfare). This is a natural benchmark that takes budgets into account, since the participants' bidding power is restricted by their budgets. $^{3}$ We compare the effective welfare of the allocation at equilibrium with the optimal effective welfare. We prove that the PoA of the proportional allocation mechanism for coarse correlated equilibria is at most $1+\phi \approx 2.618$, where $\phi$ is the golden ratio. The previously best known bound was 2.78 for a single resource and concave valuations due to [3].

Finally, we consider the polyhedral environment that was previously studied by Nguyen and Tardos in [20]. In the polyhedral environment, there exists a collection of resources and the goal is to associate each agent with a single value, representing their level of activity. Nguyen and Tardos [20] proved that pure equilibria are at least $75 \%$ efficient with concave valuations. We prove that the PoA is exactly 2 for agents with subadditive valuations. An extended abstract of the current paper appears in [7].

Related Work The efficiency of the proportional allocation mechanism has been extensively studied in the literature of network resource allocation. Besides the work mentioned above, Johari and Tsitsiklis [17] studied a more general class of scalarparametrized mechanisms and proved that the proportional allocation mechanism achieves the best PoA when the mechanism only chooses a single price. Zhang [27] and Feldman et al. [14] studied the efficiency and fairness of the proportional allocation mechanism when agents aim at maximizing non quasi-linear utilities subject to budget constraints. Correa, Schulz and Stier-Moses [9] showed a relationship in the efficiency loss between the proportional allocation mechanism and non-atomic selfish routing for not necessarily concave valuation functions.

There is a line of research studying the PoA of simple auctions for selling indivisible goods (see [2, 5, 15, 26]). Recently, Feldman et al. [13] showed tighter upper bounds for simultaneous first and second price auctions when the agents have subadditive valuations. Christodoulou et al. [6] showed matching lower bounds for

\footnotetext{
${ }^{1}$ In a simple mechanism, the agents' action space should be at most sub-doubly-exponential in $m$.

${ }^{2}$ The basic property of a scale-free mechanism is that, if every bid is scaled by the same constant, the outcome remains unchanged (we refer the reader to Section 4.3 for the complete definition).

${ }^{3}$ We illustrate the weakness of social welfare as a benchmark in the model with budgets by giving the following example. Consider a single resource and two players with valuations $h x_{1}$ and $x_{2}$, where $x_{1}$ and $x_{2}$ are the fractions allocated to player 1 and 2, respectively; $h>1$ is an arbitrarily high real value. The players' budgets are $1 / h^{2}$ and 1 , respectively. It is not hard to see that if player 1 bids $b_{1}$, player 2 's best response is $b_{2}=\sqrt{b_{1}}-b_{1}$. Therefore, for any pure Nash equilibrium $\left(b_{1}, b_{2}\right)$ the social welfare is at most $\frac{h b_{1}}{b_{1}+b_{2}}+1=h \sqrt{b_{1}}+1 \leq 2$, where for the last inequality we used the fact that player 1 's bid cannot exceed her budget, i.e., $b_{1} \leq 1 / h^{2}$. The maximum social welfare is $h$, where player 1 is allocated the whole resource, which can be arbitrarily higher than the social welfare in the equilibrium.
} 
simultaneous first price auctions, and Roughgarden [23] proved general lower bounds for the PoA of all simple auctions by using the corresponding computational or communication lower bounds of the underlying allocation problem.

\section{Preliminaries}

There are $n$ agents who compete for $m$ divisible resources with unit supply. Every agent $i \in[n]$ has a valuation function, $v_{i}:[0,1]^{m} \rightarrow \mathbb{R}_{+}$, where $[n]$ denotes the set $\{1,2, \ldots, n\}$. The valuations are monotonically non-decreasing, that is, for every two allocations, $x_{i}, x_{i}^{\prime} \in[0,1]^{m}$, where $x_{i}=\left(x_{i 1}, \ldots, x_{i m}\right), x_{i}^{\prime}=\left(x_{i 1}^{\prime}, \ldots, x_{i m}^{\prime}\right)$ and $\forall j \in[m] x_{i j} \leq x_{i j}^{\prime}$, we have $v_{i}\left(x_{i}\right) \leq v_{i}\left(x_{i}^{\prime}\right)$. We further assume that the valuations are normalized as $v_{i}((0, \ldots, 0))=0$.

In the following definition of the subadditive valuations, for any two allocation vectors $x, y \in[0,1]^{m}$, we denote the componentwise sum of $x$ and $y$ by $x+y$.

Definition 1 A function $v:[0,1]^{m} \rightarrow \mathbb{R}_{\geq 0}$ is subadditive if, for all $x, y \in[0,1]^{m}$, such that $x+y \in[0,1]^{m}$, it is $v(x+y) \leq v(x)+v(y)$.

Remark 2 Lattice submodular functions used in [26] are subadditive (see Section 4). In the case of a single variable (single resource), any concave function is subadditive; more precisely, concave functions are equivalent to lattice submodular functions in this case. However, concave functions of many variables may not be subadditive [22].

In the Bayesian setting, the valuation of each agent $i$ is drawn from a set of possible valuations $V_{i}$, according to some known probability distribution $D_{i}$. We assume that $D_{i}$ 's are independent, but not necessarily identical over the agents.

A mechanism takes the agents' bids/strategies $\mathbf{b}=\left(b_{1}, \ldots, b_{n}\right)$ as input and outputs a tuple $(\mathbf{x}, \mathbf{q})$, where $\mathbf{x}=\mathbf{x}(\mathbf{b})=\left(x_{1}, \ldots, x_{n}\right)$ specifies the allocation of resources and $\mathbf{q}=\mathbf{q}(\mathbf{b})=\left(q_{1}, \ldots, q_{n}\right)$ specifies the agents' payments. For every $i, x_{i}=\left(x_{i 1}, \ldots, x_{i m}\right)=\left(x_{i j}\right)_{j}$ denotes the allocation to agent $i$, where $x_{i j}$ is her allocation for recourse $j$, and $q_{i}$ is a real value. Every agent $i$ submits a nonnegative bid $b_{i j}$ for each resource $j$, i.e. $b_{i}=\left(b_{i 1}, \ldots, b_{i m}\right)$ is a vector. By $\mathbf{b}_{-i}=$ $\left(b_{1}, \ldots, b_{i-1}, b_{i+1}, \ldots, b_{n}\right)$ we denote the strategies of all agents except for $i$. We represent the valuations of all agents by the vector $\mathbf{v}=\mathbf{v}(\mathbf{x})=\left(v_{1}\left(x_{1}\right), \ldots, v_{n}\left(x_{n}\right)\right)$, and occasionally, instead of $v_{i}\left(x_{i}\right)$ we may use the notation $v_{i}(\mathbf{x})$, which represents agent $i$ 's valuation under the allocation $\mathbf{x}$. The utility $u_{i}$ of agent $i$ is defined as the difference between her valuation for the received allocation and her payment: $u_{i}(\mathbf{x}(\mathbf{b}), \mathbf{q}(\mathbf{b}))=u_{i}(\mathbf{b})=v_{i}\left(x_{i}(\mathbf{b})\right)-q_{i}(\mathbf{b})$. In the Bayesian setting, we use the notation $u_{i}^{v_{i}}$ in order to specify that $v_{i}$ is the valuation function of agent $i$.

The proportional allocation mechanism determines the allocation and payment for each agent $i$ as $x_{i j}=\frac{b_{i j}}{\sum_{k \in[n]} b_{k j}}$ and $q_{i}=\sum_{j \in[m]} b_{i j}$. When all agents bid 0 , the allocation can be defined arbitrarily, but consistently. 
Nash Equilibrium We use b to denote a pure strategy profile; in a more general context, we denote a strategy profile as $\mathbf{B}=\left(B_{1}, \ldots, B_{n}\right)$, where $B_{i}$ is a probability distribution over all possible pure strategies of agent $i$. In each one of the following lines, a strategy profile $\mathbf{B}$ forms the equilibrium notion on the left, if for every agent $i$ and all bids $b_{i}^{\prime}$ it satisfies the inequality on the right:

Pure Nash equilibrium: $\mathbf{B}$ chooses $\mathbf{b}$ with probability $1, u_{i}(\mathbf{b}) \geq u_{i}\left(b_{i}^{\prime}, \mathbf{b}_{-i}\right)$.

Mixed Nash equilibrium: $\mathbf{B}=\times_{i} B_{i}, \mathbb{E}_{\mathbf{b} \sim \mathbf{B}}\left[u_{i}(\mathbf{b})\right] \geq \mathbb{E}_{\mathbf{b}_{-i} \sim \mathbf{B}_{-i}}\left[u_{i}\left(b_{i}^{\prime}, \mathbf{b}_{-i}\right)\right]$.

Correlated equilibrium: $\mathbf{B}=\left(B_{i}\right)_{i}, \mathbb{E}_{\mathbf{b} \sim \mathbf{B}}\left[u_{i}(\mathbf{b}) \mid b_{i}\right] \geq \mathbb{E}_{\mathbf{b}_{-i} \sim \mathbf{B}_{-i}}\left[u_{i}\left(b_{i}^{\prime}, \mathbf{b}_{-i}\right) \mid b_{i}\right]$.

Coarse correlated equilibrium: $\mathbf{B}=\left(B_{i}\right)_{i}, \mathbb{E}_{\mathbf{b} \sim \mathbf{B}}\left[u_{i}(\mathbf{b})\right] \geq \mathbb{E}_{\mathbf{b}_{-i} \sim \mathbf{B}_{-i}}\left[u_{i}\left(b_{i}^{\prime}, \mathbf{b}_{-i}\right)\right]$.

Bayesian Nash equilibrium:

$\mathbf{B}(\mathbf{v})=\times_{i} B_{i}\left(v_{i}\right), \mathbb{E}_{\mathbf{v}_{-i}, \mathbf{b}}\left[u_{i}^{v_{i}}(\mathbf{b})\right] \geq \mathbb{E}_{\mathbf{v}_{-i}, \mathbf{b}_{-i}}\left[u_{i}\left(b_{i}^{\prime}, \mathbf{b}_{-i}\right)\right], \forall v_{i} \in V_{i}$.

The first four classes of equilibria are in increasing order of inclusion. Moreover, any mixed Nash equilibrium is also a Bayesian Nash equilibrium.

Price of Anarchy (PoA) Our global objective is to maximize the sum of the agents' valuations for their received allocations, i.e., to maximize the social welfare $\mathrm{SW}(\mathbf{x})=$ $\sum_{i \in[n]} v_{i}\left(x_{i}\right)$. Given the valuations, $\mathbf{v}$, of all agents, there exists an optimal allocation $\mathbf{o}^{\mathbf{v}}=\mathbf{o}=\left(o_{1}, \ldots, o_{n}\right)$, such that $\mathrm{SW}(\mathbf{0})=\max _{\mathbf{x}} \mathrm{SW}(\mathbf{x})$. By $o_{i}=\left(o_{i 1}, \ldots, o_{i m}\right)$ we denote the optimal allocation to agent $i$. For simplicity, we use $\mathrm{SW}(\mathbf{b})$ and $v_{i}(\mathbf{b})$ instead of $\mathrm{SW}(\mathbf{x}(\mathbf{b}))$ and $v_{i}\left(x_{i}(\mathbf{b})\right)$ whenever the allocation rule $\mathbf{x}$ is clear from the context. We also use shorter notation for expectations, e.g., we use $\mathbb{E}_{\mathbf{v}}$ instead of $\mathbb{E}_{\mathbf{v} \sim \mathbf{D}}, \mathbb{E}\left[u_{i}(\mathbf{b})\right]$ instead of $\mathbb{E}_{\mathbf{b} \sim \mathbf{B}}\left[u_{i}(\mathbf{b})\right]$ and $u(\mathbf{B})$ instead of $\mathbb{E}_{\mathbf{b} \sim \mathbf{B}}[u(\mathbf{b})]$ whenever $\mathbf{D}$ and $\mathbf{B}$ are clear from the context.

Definition 3 Let $\mathcal{I}([n],[m], \mathbf{v})$ be the set of all instances, i.e., $\mathcal{I}([n],[m], \mathbf{v})$ includes the instances for every set of agents and resources and any possible valuations that the agents might have for the resources. We define the pure, mixed, correlated, coarse correlated and Bayesian Price of Anarchy as

$$
\operatorname{PoA}=\max _{I \in \mathcal{I}} \max _{\mathbf{B} \in \mathcal{E}(I)} \frac{\mathbb{E}_{\mathbf{v}}[\mathrm{SW}(\mathbf{o})]}{\mathbb{E}_{\mathbf{v}, \mathbf{b} \sim \mathbf{B}}[\mathrm{SW}(\mathbf{b})]},
$$

where $\mathcal{E}(I)$ is the set of pure Nash, mixed Nash, correlated, coarse correlated or Bayesian Nash equilibria for the specific instance $I \in \mathcal{I}$, respectively. ${ }^{4}$

Budget Constraints We also consider the setting where agents are budgetconstrained. That is, the payment of each agent $i$ cannot be higher than $c_{i}$, where $c_{i}$ is a non-negative value denoting agent $i$ 's budget. Following $[3,10,26]$, we use Effective Welfare as the benchmark: $\operatorname{EW}(\mathbf{x})=\sum_{i} \min \left\{v_{i}\left(x_{i}\right), c_{i}\right\}$. In addition, for any randomized allocation $\mathbf{x}$, the expected effective welfare is defined as: $\mathbb{E}_{\mathbf{x}}[\mathrm{EW}(\mathbf{x})]=\sum_{i} \min \left\{\mathbb{E}_{\mathbf{x}}\left[v_{i}\left(x_{i}\right)\right], c_{i}\right\}$ and in the Bayesian setting $\mathbb{E}_{\mathbf{v}}[\operatorname{EW}(\mathbf{x}(\mathbf{v}))]=$ $\sum_{i} \mathbb{E}_{v_{i}, c_{i}}\left[\min \left\{\mathbb{E}_{\mathbf{v}_{-i}, \mathbf{c}_{-i}}\left[v_{i}\left(x_{i}(\mathbf{v})\right)\right], c_{i}\right\}\right]$.

\footnotetext{
${ }^{4}$ The expectation over $\mathbf{v}$ is only needed for the definition of Bayesian PoA.
} 


\section{Concave Valuations}

In this section, we show that, for concave valuations on multiple resources, Bayesian equilibria can be arbitrarily inefficient. More precisely, we prove that the Bayesian PoA is $\Omega(\sqrt{m})$ in contrast to the constant bound for pure equilibria [16]. Therefore, there is a big gap between complete and incomplete information settings. We state our main theorem in this section as follows.

Theorem 4 When valuations are concave the PoA of the proportional allocation mechanism for Bayesian equilibria is at least $\frac{\sqrt{m}}{2}$.

Proof We consider an instance with $m$ resources and 2 agents with the following concave valuations. $v_{1}(\mathbf{x})=\min _{j}\left\{x_{1 j}\right\}$ and $v_{2}(\mathbf{x})$ is drawn from a distribution $D_{2}$, such that some resource $j \in[m]$ is chosen uniformly at random and then $v_{2}(\mathbf{x})=$ $x_{2 j} / \sqrt{m}$. Let $\delta=1 /(\sqrt{m}+1)^{2}$. We claim that $\mathbf{b}(\mathbf{v})=\left(b_{1}, b_{2}\left(v_{2}\right)\right)$ is a pure Bayesian Nash equilibrium, where $\forall j \in[m], b_{1 j}=\sqrt{\delta / m}-\delta$ and, for $j^{*}$ being the resource chosen by $D_{2}, b_{2} j^{*}\left(v_{2}\right)=\delta$ whereas, for all $j \neq j^{*}, b_{2 j}=0$.

Under this bidding profile, agent 1 bids the same value for all resources and agent 2 only bids positive value for a single resource associated with her valuation. Suppose that agent 2 has positive valuation for resource $j$, i.e., $v_{2}(\mathbf{x})=x_{2 j} / \sqrt{m}$. Then, the rest $m-1$ resources are allocated to agent 1 and agents are competing for resource $j$. Bidder 2 has no reason to bid positively for any other resource. If she bids any value $b_{2 j}^{\prime}$ for resource $j$, her utility would be $u_{2}\left(\mathbf{b}_{1}, b_{2 j}^{\prime}\right)=\frac{1}{\sqrt{m}} \frac{b_{2 j}^{\prime}}{b_{1 j}+b_{2 j}^{\prime}}-b_{2 j}^{\prime}$, which is maximized for $b_{2 j}^{\prime}=\sqrt{\frac{b_{1 j}}{\sqrt{m}}}-b_{1 j}$. For $b_{1 j}=\sqrt{\delta / m}-\delta$, the utility of agent 2 is maximized for $b_{2 j}^{\prime}=1 /(\sqrt{m}+1)^{2}=\delta$ by simple calculations.

Since $v_{1}(\mathbf{x})$ equals the minimum of $\mathbf{x}$ 's components, agent 1's valuation is completely determined by the allocation of resource $j$. So the expected utility of agent 1 under $\mathbf{b}$ is $\mathbb{E}_{v_{2}}\left[u_{1}(\mathbf{b})\right]=\frac{\sqrt{\delta / m}-\delta}{\sqrt{\delta / m}-\delta+\delta}-m(\sqrt{\delta / m}-\delta)=(1-\sqrt{m \delta})^{2}=\frac{1}{(\sqrt{m}+1)^{2}}=\delta$. Suppose now that agent 1 deviates to $b_{1}^{\prime}=\left(b_{11}^{\prime}, \ldots, b_{1 m}^{\prime}\right)$.

$$
\begin{aligned}
\underset{v_{2}}{\mathbb{E}}\left[u_{1}\left(b_{1}^{\prime}, b_{2}\right)\right] & =7 \frac{1}{m} \sum_{j} \frac{b_{1 j}^{\prime}}{b_{1 j}^{\prime}+\delta}-\sum_{j} b_{1 j}^{\prime}=\frac{1}{m} \sum_{j}\left(\frac{b_{1 j}^{\prime}}{b_{1 j}^{\prime}+\delta}-m \cdot b_{1 j}^{\prime}\right) \\
& \leq \frac{1}{m} \sum_{j}\left(\frac{\sqrt{\delta / m}-\delta}{\sqrt{\delta / m}}-m \cdot(\sqrt{\delta / m}-\delta)\right) \\
& =\frac{1}{m} \sum_{j}(1-2 \sqrt{m \cdot \delta}+m \cdot \delta)=\frac{1}{m} \sum_{j}(1-\sqrt{m \cdot \delta})^{2} \\
& =\frac{1}{m} \sum_{j}\left(\frac{1}{\sqrt{m}+1}\right)^{2}=\delta=\underset{v_{2}}{\mathbb{E}}\left[u_{1}(\mathbf{b})\right] .
\end{aligned}
$$

The inequality comes from the fact that $\frac{b_{1 j}^{\prime}}{b_{1 j}^{\prime}+\delta}-m \cdot b_{1 j}^{\prime}$ is maximized for $b_{1 j}^{\prime}=$ $\sqrt{\delta / m}-\delta$. So we conclude that $\mathbf{b}$ is a Bayesian equilibrium. 
Finally, we compute the PoA. The expected social welfare under $\mathbf{b}$ is $\mathbb{E}_{v_{2}}[\mathrm{SW}(\mathbf{b})]=\frac{\sqrt{\delta / m}-\delta}{\sqrt{\delta / m}-\delta+\delta}+\frac{1}{\sqrt{m}} \frac{\delta}{\sqrt{\delta / m}-\delta+\delta}=1-\sqrt{m \delta}+\sqrt{\delta}=\frac{2}{\sqrt{m}+1}<\frac{2}{\sqrt{m}}$. The optimal social welfare is 1 by allocating to agent 1 all resources. So, $\mathrm{PoA} \geq \frac{\sqrt{m}}{2}$.

\section{Subadditive Valuations}

In this section, we focus on agents with subadditive valuations. We prove that the proportional allocation mechanism is at least $50 \%$ efficient for coarse correlated equilibria and Bayesian Nash equilibria, i.e., PoA $\leq 2$. We further show that this bound is tight and cannot be improved by any simple or scale-free mechanism. Before proving our PoA bounds, we show that the class of subadditive functions is a superclass of lattice submodular functions.

Proposition 5 Any lattice submodular function $v$ defined on $[0,1]^{m}$ is subadditive.

Proof It has been shown in [26] that, for any lattice submodular function $v(x)$, $\frac{\partial^{2} v(x)}{\left(\partial x_{j}\right)^{2}} \leq 0$ and $\frac{\partial^{2} v(x)}{\partial x_{j} \partial x_{j^{\prime}}} \leq 0$. So, the function $\frac{\partial v}{\partial x_{j}}(x)$ is non-increasing monotone for each coordinate $x_{j^{\prime}}$. It suffices to prove that for any $\mathbf{x}, \mathbf{y} \in[0,1]^{m}, v(\mathbf{x}+\mathbf{y})-v(\mathbf{y}) \leq$ $v(\mathbf{x})-v(\mathbf{0})$. Let $\mathbf{z}^{k}$ be the vector that $z_{j}^{k}=y_{j}$ if $j \leq k$ and $x_{j}+y_{j}$ otherwise. Note that $\mathbf{z}^{0}=\mathbf{x}+\mathbf{y}$ and $\mathbf{z}^{m}=\mathbf{y}$. Similarly, we define $\mathbf{w}^{k}$ to be the vector that $\mathbf{w}_{j}^{k}=0$ if $j \leq k$ and $x_{j}$ otherwise. It is easy to see that $\mathbf{z}^{k} \geq \mathbf{w}^{k}$ for all $k \in[m]$. So, we have

$$
\begin{aligned}
& v(\mathbf{x}+\mathbf{y})-v(\mathbf{y})=\sum_{j \in[m]} v\left(\mathbf{z}^{j-1}\right)-v\left(\mathbf{z}^{j}\right)=\sum_{j \in[m]} \int_{y_{j}}^{x_{j}+y_{j}} \frac{\partial v}{\partial x_{j}}\left(t_{j} ; \mathbf{z}_{-j}^{j}\right) d t_{j} \\
\leq & \sum_{j \in[m]} \int_{y_{j}}^{x_{j}+y_{j}} \frac{\partial v}{\partial x_{j}}\left(t_{j}-y_{j} ; \mathbf{z}_{-j}^{j}\right) d t_{j} \leq \sum_{j \in[m]} \int_{0}^{x_{j}} \frac{\partial v}{\partial x_{j}}\left(s_{j} ; \mathbf{w}_{-j}^{j}\right) d s_{j}=v(\mathbf{x})-v(\mathbf{0}) .
\end{aligned}
$$

The second equality is due to the definition of partial derivative and the inequalities is due to the monotonicity of $\frac{\partial v}{\partial x_{j}}(x)$.

\subsection{Upper Bound}

A common approach to prove PoA upper bounds is to find a deviation with proper utility bounds and then use the definition of Nash equilibrium to bound agents' utilities at equilibrium. The bidding strategy described in the following lemma is for this purpose.

Lemma 6 Let $\mathbf{v}$ be any subadditive valuation profile and $\mathbf{B}$ be some randomized bidding profile. For any agent $i$, there exists a randomized bidding strategy, $a_{i}\left(\mathbf{v}, \mathbf{B}_{-i}\right)$, such that:

$$
\sum_{i} u_{i}\left(a_{i}\left(\mathbf{v}, \mathbf{B}_{-i}\right), \mathbf{B}_{-i}\right) \geq \frac{1}{2} \sum_{i} v_{i}\left(o_{i}^{\mathbf{v}}\right)-\sum_{i} \sum_{j} \underset{\mathbf{b} \sim \mathbf{B}}{\mathbb{E}}\left[b_{i j}\right]
$$


Proof Let $p_{i j}$ be the sum of the bids of all agents except $i$ on resource $j$, i.e., $p_{i j}=$ $\sum_{k \neq i} b_{k j}$. Note that $p_{i j}$ is a random variable that depends on $\mathbf{b}_{-i} \sim \mathbf{B}_{-i}$. Let $P_{i}$ be the propability distribution of $p_{i}=\left(p_{i j}\right)_{j}$. Inspired by [13], we consider the bidding strategy $a_{i}\left(\mathbf{v}, \mathbf{B}_{-i}\right)=\left(o_{i j}^{\mathbf{v}} \cdot b_{i j}^{\prime}\right)_{j}$, where $b_{i}^{\prime} \sim P_{i}$. Then, $u_{i}\left(a_{i}\left(\mathbf{v}, \mathbf{B}_{-i}\right), \mathbf{B}_{-i}\right)$ is

$$
\begin{aligned}
& \left.\underset{b_{i}^{\prime} \sim P_{i} p_{i} \sim P_{i}}{\mathbb{E}} \underset{v_{i}}{\mathbb{E}}\left[\left(\frac{o_{i j}^{\mathbf{v}} b_{i j}^{\prime}}{o_{i j}^{\mathbf{v}} b_{i j}^{\prime}+p_{i j}}\right)_{j}\right)-o_{i}^{\mathbf{v}} \cdot b_{i}^{\prime}\right] \\
& \geq \frac{1}{2} \cdot \underset{p_{i} \sim P_{i} b_{i}^{\prime} \sim P_{i}}{\mathbb{E}}\left[v_{i}\left(\left(\frac{o_{i j}^{\mathbf{v}} b_{i j}^{\prime}}{o_{i j}^{\mathbf{v}} b_{i j}^{\prime}+p_{i j}}+\frac{o_{i j}^{\mathbf{v}} p_{i j}}{o_{i j}^{\mathbf{v}} p_{i j}+b_{i j}^{\prime}}\right){ }_{j}\right)\right]-\underset{p_{i} \sim P_{i}}{\mathbb{E}}\left[o_{i}^{\mathbf{v}} \cdot p_{i}\right] \\
& \geq \frac{1}{2} \cdot \underset{p_{i} \sim P_{i} b_{i}^{\prime} \sim P_{i}}{\mathbb{E}}\left[v_{i}\left(\left(\frac{o_{i j}^{\mathbf{v}}\left(b_{i j}^{\prime}+p_{i j}\right)}{b_{i j}^{\prime}+p_{i j}}\right)\right)\right]-\underset{p_{i} \sim P_{i}}{\mathbb{E}}\left[o_{i}^{\mathbf{v}} \cdot p_{i}\right] \\
& =\frac{1}{2} \cdot v_{i}\left(o_{i}^{\mathbf{v}}\right)-\sum_{j} \sum_{k \neq i} \underset{\mathbf{b} \sim \mathbf{B}}{\mathbb{E}}\left[o_{i j}^{\mathbf{v}} \cdot b_{k j}\right] \\
& =\frac{1}{2} \cdot v_{i}\left(o_{i}^{\mathbf{v}}\right)-\sum_{j} o_{i j}^{\mathbf{v}} \sum_{k \neq i} \underset{\mathbf{b} \sim \mathbf{B}}{\mathbb{E}}\left[b_{k j}\right] \\
& \geq \frac{1}{2} \cdot v_{i}\left(o_{i}^{\mathbf{v}}\right)-\sum_{j} o_{i j}^{\mathbf{v}} \sum_{k \in[n]} \underset{\mathbf{b} \sim \mathbf{B}}{\mathbb{E}}\left[b_{k j}\right] .
\end{aligned}
$$

The first inequality follows by swapping $p_{i j}$ and $b_{i j}^{\prime}$ and using the subadditivity of $v_{i}$. The second inequality comes from the fact that $o_{i j}^{\mathbf{v}} \leq 1$. The lemma follows by summing up over all agents and the fact that $\sum_{i \in[n]} o_{i j}^{\mathbf{v}}=1$.

Theorem 7 The coarse correlated PoA of the proportional allocation mechanism with subadditive agents is at most 2.

Proof Let $\mathbf{B}$ be any coarse correlated equilibrium (note that $\mathbf{v}$ is fixed). By Lemma 6 and the definition of the coarse correlated equilibrium we have

$$
\sum_{i} u_{i}(\mathbf{B}) \geq \sum_{i} u_{i}\left(a_{i}\left(\mathbf{v}, \mathbf{B}_{-i}\right), \mathbf{B}_{-i}\right) \geq \frac{1}{2} \sum_{i} v_{i}\left(o_{i}\right)-\sum_{i} \sum_{j} \mathbb{E}\left[b_{i j}\right] .
$$

By rearranging terms, $\mathrm{SW}(\mathbf{B})=\sum_{i} u_{i}(\mathbf{B})+\sum_{i} \sum_{j} \mathbb{E}\left[b_{i j}\right] \geq \frac{1}{2} \cdot \mathrm{SW}(\mathbf{o})$.

Theorem 8 The Bayesian PoA of the proportional allocation mechanism with subadditive agents is at most 2.

Proof Let $\mathbf{B}$ be any Bayesian Nash Equilibrium and let $v_{i} \sim D_{i}$ be the valuation of each agent $i$ drawn independently from $D_{i}$. We denote by $\mathbf{C}=\left(C_{1}, C_{2}, \ldots, C_{n}\right)$ the 
bidding distribution in $\mathbf{B}$ which includes the randomness of both the bidding strategy $\mathbf{b}$ and of the valuations $\mathbf{v}$. The utility of agent $i$ with valuation $v_{i}$ can be expressed by $u_{i}\left(\mathbf{B}_{i}\left(v_{i}\right), \mathbf{C}_{-i}\right)$. It should be noted that $\mathbf{C}_{-i}$ does not depend on some particular $\mathbf{v}_{-i}$, but merely on $\mathbf{D}_{-i}$ and $\mathbf{B}_{-i}$. For any agent $i$ and any subadditive valuation $v_{i} \in V_{i}$, consider the deviation $a_{i}\left(v_{i} ; \mathbf{w}_{-i}, \mathbf{C}_{-i}\right)$ as defined in Lemma 6, where $\mathbf{w}_{-i} \sim \mathbf{D}_{-i}$. By the definition of the Bayesian Nash equilibrium we obtain

$$
\underset{\mathbf{v}_{-i}}{\mathbb{E}}\left[u_{i}^{v_{i}}\left(\mathbf{B}_{i}\left(v_{i}\right), \mathbf{B}_{-i}\left(\mathbf{v}_{-i}\right)\right)\right]=u_{i}^{v_{i}}\left(\mathbf{B}_{i}\left(v_{i}\right), \mathbf{C}_{-i}\right) \geq \underset{\mathbf{w}_{-i}}{\mathbb{E}}\left[u_{i}^{v_{i}}\left(a_{i}\left(v_{i} ; \mathbf{w}_{-i}, \mathbf{C}_{-i}\right), \mathbf{C}_{-i}\right)\right] .
$$

By taking expectation over $v_{i}$ and summing up over all agents

$$
\begin{aligned}
& \sum_{i} \underset{\mathbf{v}}{\mathbb{E}}\left[u_{i}(\mathbf{B}(\mathbf{v}))\right] \geq \sum_{i} \underset{v_{i}, \mathbf{w}_{-i}}{\mathbb{E}}\left[u_{i}^{v_{i}}\left(a_{i}\left(v_{i} ; \mathbf{w}_{-i}, \mathbf{C}_{-i}\right), \mathbf{C}_{-i}\right)\right] \\
= & \underset{\mathbf{v}}{\mathbb{E}}\left[\sum_{i} u_{i}^{v_{i}}\left(a_{i}\left(\mathbf{v}, \mathbf{C}_{-i}\right), \mathbf{C}_{-i}\right)\right] \geq \frac{1}{2} \cdot \sum_{i} \underset{\mathbf{v}}{\mathbb{E}}\left[v_{i}\left(o_{i}^{\mathbf{v}}\right)\right]-\sum_{i} \sum_{j} \mathbb{E}\left[b_{i j}\right] .
\end{aligned}
$$

So, $\mathbb{E}_{\mathbf{v}}[\operatorname{SW}(\mathbf{B}(\mathbf{v}))]=\sum_{i} \mathbb{E}_{\mathbf{v}}\left[u_{i}(\mathbf{B}(\mathbf{v}))\right]+\sum_{i} \sum_{j} \mathbb{E}\left[b_{i j}\right] \geq \frac{1}{2} \cdot \mathbb{E}_{\mathbf{v}}\left[\operatorname{SW}\left(\mathbf{o}^{\mathbf{v}}\right)\right]$.

As we show next, the previous upper bounds of Theorems 7 and 8 are tight even for the pure Nash equilibrium and a single resource.

Theorem 9 The pure PoA of the proportional allocation mechanism with subadditive agents is at least 2.

Proof We consider a game with only two agents and a single resource. The valuation of the first agent is $v_{1}(x)=1+\epsilon \cdot x$, for some $\epsilon<1$, if $x<1$ and $v_{1}(x)=2$ if $x=1$. The valuation of the second agent is $\epsilon \cdot x$. One can easily verify that these two functions are subadditive and the optimal social welfare is 2 . Consider the bidding strategies $b_{1}=b_{2}=\frac{\epsilon}{4}$. The utility of agent 1 , when she bids $b_{1}^{\prime}$ and agent 2 bids $\frac{\epsilon}{4}$, is given by $1+\epsilon \cdot \frac{b_{1}^{\prime}}{b_{1}^{\prime}+\epsilon / 4}-b_{1}^{\prime}$, which is maximized for $b_{1}^{\prime}=\frac{\epsilon}{4}$. The utility of agent 2 , when she bids $b_{2}^{\prime}$ and agent 1 bids $\frac{\epsilon}{4}$, is $\epsilon \cdot \frac{b_{2}^{\prime}}{b_{2}^{\prime}+\epsilon / 4}-b_{2}^{\prime}$, which is also maximized when $b_{2}^{\prime}=\frac{\epsilon}{4}$. So $\left(b_{1}, b_{2}\right)$ is a pure Nash Equilibrium with social welfare $1+\epsilon$. Therefore, the PoA converges to 2 when $\epsilon$ goes to 0 .

\subsection{Simple Mechanisms Lower Bound}

Now we show a lower bound that applies to all simple mechanisms, where the bidding space has size (at most) sub-doubly-exponential in $m$. More specifically, we apply the general framework of Roughgarden [23] for showing lower bounds on the price of anarchy for all simple mechanisms via communication complexity reductions with respect to the underlying optimization problem. In our setting, the problem is to maximize the social welfare by allocating divisible resources to agents with 
subadditive valuations. We proceed by proving a communication lower bound for this problem in the following lemma.

Lemma 10 For any constant $\varepsilon>0$, any $(2-\varepsilon)$-approximation (non-deterministic) algorithm for maximizing social welfare in resource allocation problem with subadditive valuations requires an exponential amount of communication.

Proof We prove this lemma by reducing the communication lower bound for combinatorial auctions with general valuations (Theorem 3 of [21]) to our setting (see also [11] for a reduction to combinatorial auctions with subadditive agents).

Nisan [21] used an instance with $n$ players and $m$ items, with $n<m^{1 / 2-\varepsilon}$. Each player $i$ is associated with a set $T_{i}$, with $\left|T_{i}\right|=t$ for some $t>0$. At every instance of this problem, the players' valuations are determined by sets $I_{i}$ of bundles, where $I_{i} \subseteq T_{i}$ for every $i$. Given $I_{i}$, player $i$ 's valuation on some subset $S$ of items is $v_{i}(S)=1$, if there exists some $R \in I_{i}$ such that $R \subseteq S$, otherwise $v_{i}(S)=0$. In [21], it was shown that distinguishing between instances with optimal social welfare of $n$ and 1 requires $t$ bits of communication. By choosing $t$ exponential in $m$ their theorem follows.

We prove the lemma by associating any valuation $v$ of the above combinatorial auction problem to some appropriate subadditive valuation $v^{\prime}$ for our setting. For any player $i$ and any fractional allocation $\mathbf{x}=\left(x_{1}, \ldots, x_{m}\right)$, let $A_{x_{i}}=\left\{j \mid x_{i j}>\frac{1}{2}\right\}$. We define $v_{i}^{\prime}\left(x_{i}\right)=v_{i}\left(A_{x_{i}}\right)+1$, if $x_{i} \neq \mathbf{0}$ and $v_{i}^{\prime}\left(x_{i}\right)=0$, otherwise. It is easy to verify that $v_{i}^{\prime}$ is subadditive. Notice that $v_{i}^{\prime}(x)=2$ only if there exists $R \in I_{i}$ such that player $i$ is allocated a fraction higher than $1 / 2$ for every resource in $R$. The value $1 / 2$ is chosen such that no two players are assigned more than that fraction from the same resource. This corresponds to the constraint of an allocation in the combinatorial auction where no item is allocated to two players.

Therefore, in the divisible goods allocation problem, distinguishing between instances where the optimal social welfare is $2 n$ and $n+1$ is equivalent to distinguishing between instances where the optimal social welfare is $n$ and 1 in the corresponding combinatorial auction and hence requires exponential, in $m$, number of communication bits.

The PoA lower bound follows the general reduction described in [23].

Theorem 11 The PoA of $\epsilon$-mixed Nash equilibria ${ }^{5}$ of every simple mechanism, when agents have subadditive valuations, is at least 2.

Remark 12 This result holds only for $\epsilon$-mixed Nash equilibria. By considering exact Nash equilibria we show a lower bound for all scale-free mechanisms in the following section.

\footnotetext{
${ }^{5} \mathrm{~A}$ bidding profile $\mathbf{B}=\times_{i} B_{i}$ is called $\epsilon$-mixed Nash equilibrium if, for every agent $i$ and all bids $b_{i}^{\prime}$, $\mathbb{E}_{\mathbf{b} \sim \mathbf{B}}\left[u_{i}(\mathbf{b})\right] \geq \mathbb{E}_{\mathbf{b} \sim \mathbf{B}}\left[u_{i}\left(b_{i}^{\prime}, \mathbf{b}_{-i}\right)\right]-\epsilon$.
} 


\subsection{Scale-free Mechanisms Lower Bound}

Here we prove a tight lower bound for all scale-free mechanisms including the proportional allocation mechanism. A mechanism $(\mathbf{x}, \mathbf{q})$ is said to be scale-free if a) for every agent $i$, resource $j$ and constant $c>0, x_{i j}\left(c \cdot \mathbf{b}_{j}\right)=x_{i j}\left(\mathbf{b}_{j}\right)$; moreover, for a fixed $\mathbf{b}_{-i}, x_{j i}(\cdot)$ is non-decreasing and positive whenever $b_{i j}$ is positive, b) the payment for agent $i$ depends only on her bids $b_{i}=\left(b_{i j}\right)_{j}$ and is equal to $\sum_{j \in[m]} q_{i}\left(b_{i j}\right)$, where $q_{i}(\cdot)$ is non-decreasing, continuous, normalized $\left(q_{i}(0)=0\right)$, and there always exists a bid $b_{i j}$ such that $q_{i}\left(b_{i j}\right)>0$.

Theorem 13 The mixed PoA of scale-free mechanisms when agents have subbaditive valuations, is at least 2.

Proof Given a mechanism $(\mathbf{x}, \mathbf{q})$, we construct an instance with 2 agents and $m$ resources. Let $V$ be a positive value such that $V / m$ is in the range of both $q_{1}$ and $q_{2}$. This can be always done due to our assumptions on $q_{i}$. Let $T_{1}$ and $T_{2}$ be the values such that $q_{1}\left(T_{1}\right)=q_{2}\left(T_{2}\right)=V / m$. W.l.o.g. we assume that $T_{1} \geq T_{2}$. By monotonicity of $q_{1}, q_{1}\left(T_{2}\right) \leq V / m$. Pick an arbitrary value $a \in(0,1)$, and let $h_{1 j}=x_{1 j}(a, a)$ and $h_{2 j}=x_{2 j}(a, a)$, for every $j \in[m]$. By the assumption that $x_{i j}\left(\mathbf{b}_{j}\right)>0$ for $b_{i j}>0$ we have $h_{1 j}, h_{2 j} \in(0,1)$ for all $j \in[m]$. Let $v=V / \sqrt{m}$. We define the agents' valuations as:

$v_{1}(\mathbf{x})=\left\{\begin{array}{ll}0, & \text { if } \forall j \in[m], x_{1 j}=0, \\ v, & \text { if } \forall j x_{1 j}<h_{1 j}, \exists k x_{1 k}>0 \\ 2 v, & \text { otherwise }\end{array} \quad v_{2}(\mathbf{x})= \begin{cases}0, & \text { if } \forall j \in[m], x_{1 j}=0 \\ V, & \text { if } \exists j x_{1 j}<h_{2 j}, \exists k x_{1 k}>0 \\ 2 V, & \text { otherwise }\end{cases}\right.$

We claim that the following mixed strategy profile $\mathbf{B}$ is a Nash equilibrium. Agent 1 picks resource $l$ uniformly at random and bids $b_{1 l}=y$, and $b_{1 j}=0$, for $j \neq l$, where $y$ is a random variable drawn by the cumulative distribution $G(y)=$ $\frac{m q_{2}(y)}{V}, y \in\left[0, T_{2}\right]$. Agent 2 bids $b_{2 j}=z$ for every resource $j$, where $z$ is a random variable drawn from $F(z)$, defined as $F(z)=\frac{v-q_{1}\left(T_{2}\right)+q_{1}(z)}{v}, \quad z \in\left[0, T_{2}\right]$. Recall that $v=V / \sqrt{m}$ and $q_{1}\left(T_{2}\right) \leq V / m$. Therefore, $v-q_{1}\left(T_{2}\right) \geq 0$ and thus $F(0) \geq 0$. Notice that $G(\cdot)$ and $F(\cdot)$ are valid CDFs, due to monotonicity of $q_{i}(\cdot)$ and also $G\left(T_{2}\right)=1, F\left(T_{2}\right)=1$. Since $q_{i}(\cdot)$ is continuous, $G(y)$ and $F(y)$ are continuous in $(0, \infty)$ and therefore both functions have no mass point in any $y \neq 0$. We assume that if both agents bid 0 for some resource, agent 2 is allocated the whole resource. We are ready to show that $\mathbf{B}$ is a Nash equilibrium. For the following arguments bare in mind that $G\left(T_{2}\right)=1, F\left(T_{2}\right)=1$ and $G(0)=0$.

If agent 1 bids any $y$ in the range $\left(0, T_{2}\right.$ ] for a single resource $j$ and zero for the rest, then she gets allocation of at least $h_{1 j}$ (that she values for $2 v$ ), only if $y \geq z$, which happens with probability $F(y)$. This holds due to monotonicity of $x_{1 j}(\cdot)$ with respect to $y$. Otherwise her value is $v$. Therefore, her expected valuation is $v+F(y) v$. So, for every $y \in\left(0, T_{2}\right]$ her expected utility is $v+F(y) v-q_{1}(y)=2 v-q_{1}\left(T_{2}\right)$. If agent 1 picks $y$ according to $G(y)$, her utility is still $2 v-q_{1}\left(T_{2}\right)$, since she bids 0 
with zero probability. Suppose agent 1 bids $\mathbf{y}=\left(y_{1}, \ldots, y_{m}\right), y_{j} \in\left[0, T_{2}\right]$ for every $j$, with at least two positive bids, and w.l.o.g., assume $y_{1}=\max _{j} y_{j}$. If $z>y_{1}$, agent 1 has value $v$ for the allocation she receives and if $z \leq y_{1}$, agent 1 has value $2 v$. But in both cases she pays more than $q_{1}\left(y_{1}\right)$ and so, this strategy is dominated by the strategy of bidding $y_{1}$ for the first resource and zero for the rest. Bidding greater than $T_{2}$ for any resource is dominated by the strategy of bidding exactly $T_{2}$ for that resource.

If agent 2 bids $z \in\left[0, T_{2}\right]$ for all resources, she gets an allocation of at least $h_{2 j}$, for every $j \in[m]$, with probability $G(z)$ (due to monotonicity of $x_{2 j}(\cdot)$ with respect to $z$ and to the tie breaking rule). So, her expected utility is $V+G(z) V-$ $m q_{2}(z)=V$. Bidding greater than $T_{2}$ for any resource is dominated by bidding exactly $T_{2}$ for this resource. Suppose that agent 2 bids any $\mathbf{z}=\left(z_{1}, \ldots z_{m}\right)$, with $z_{j} \in\left[0, T_{2}\right]$ for every $j$, then, since agent 1 bids positively for any resource with probability $1 / m$, agent's 2 expected utility is $\frac{1}{m} \sum_{j}\left(V+G\left(z_{j}\right) V-\sum_{k} q_{2}\left(z_{k}\right)\right)=$ $\frac{1}{m} \sum_{j}\left(V+m q_{2}\left(z_{j}\right)-\sum_{k} q_{2}\left(z_{k}\right)\right)=\frac{1}{m}\left(m V+m \sum_{j} q_{2}\left(z_{j}\right)-m \sum_{k} q_{2}\left(z_{k}\right)\right)=$ $V$. So, $\mathbf{B}$ is Nash equilibrium.

Therefore, it is sufficient to bound the expected social welfare in B. First notice that the valuation of agents 1 and 2 are, according to the definition, at most $2 v$ and $2 \mathrm{~V}$, respectively. Agent 1 bids 0 with zero probability, so, with probability 1 if agent 2 bids 0 , there exists some resource $j$ from which she receives a fraction less than $h_{2 j}$. Her valuation in that case is $V$, meaning that in expectation the valuation of agent 2 is at most $2 V-F(0) \cdot V$. Agent 2 bids 0 with probability $F(0)=1-\frac{q_{1}\left(T_{2}\right)}{v} \geq 1-\frac{V}{m v}=$ $1-\frac{1}{\sqrt{m}}$. Hence, $\mathbb{E}[\mathrm{SW}(\mathbf{B})] \leq 2 V-F(0) \cdot V+2 v \leq 2 V-V\left(1-\frac{1}{\sqrt{m}}\right)+2 V \frac{1}{\sqrt{m}}=$ $V\left(1+\frac{3}{\sqrt{m}}\right)$. On the other hand, the social welfare in the optimum allocation is $2(V+v)=2 V\left(1+\frac{1}{\sqrt{m}}\right)$ (agent 1 is allocated $h_{1 j}$ proportion from some resource $j$ and the rest is allocated to agent 2). We conclude that $P o A \geq 2 \frac{\left(1+\frac{1}{\sqrt{m}}\right)}{\left(1+\frac{3}{\sqrt{m}}\right)}$ which, for large $m$, converges to 2 .

\section{Budget Constraints}

In this section, we switch to scenarios where agents have budget constraints. We use as a benchmark the effective welfare similarly to [3, 10,26]. We compare the effective welfare of the allocation at equilibrium with the optimal effective welfare. We prove an upper bound of $\phi+1 \approx 2.618$ for coarse correlated equilibria when bidders have subadditive valuations and compete for many resources, where $\phi=\frac{\sqrt{5}+1}{2}$ is the golden ratio. This improves the previously known 2.78 upper bound in [3] for a single resource and concave valuations.

To prove this upper bound we use the fact that in the equilibrium there is no profitable unilateral deviation and in particular, the utility of agent $i$ obtained by any 
pure deviating bid $a_{i}$ should be bounded by her budget $c_{i}$, i.e., $\sum_{j \in[m]} a_{i j} \leq c_{i}$. We define $v^{c}$ to be the valuation $v$ suppressed by the budget $c$, i.e., $v^{c}(x)=\min \{v(x), c\}$. Note that $v^{c}$ is also subadditive since $v$ is subadditive. For a fixed pair $(\mathbf{v}, \mathbf{c})$, let $\mathbf{0}=\left(o_{1}, \ldots, o_{n}\right)$ be the allocation that maximizes the effective welfare. For a fixed agent $i$ and a vector of bids $\mathbf{b}_{-i}$, we define the vector $p_{i}=\left(p_{i j}\right)_{j}$ where $p_{i j}=\sum_{k \neq i} b_{k j}$ for all $j \in[m]$. We first show the existence of a proper deviation.

Lemma 14 For any subadditive agent $i$, and any randomized bidding profile $\mathbf{B}$, there exists a randomized bid $a_{i}\left(\mathbf{B}_{-i}\right)$, such that for any $\lambda \geq 1$, it is

$$
u_{i}\left(a_{i}\left(\mathbf{B}_{-i}\right), \mathbf{B}_{-i}\right) \geq \frac{v_{i}^{c_{i}}\left(o_{i}\right)}{\lambda+1}-\frac{\sum_{j \in[m]} \sum_{k \in[n]} o_{i j} \mathbb{E}\left[b_{k j}\right]}{\lambda} .
$$

Moreover, for any pure strategy $\hat{a}_{i}$ in the support of $a_{i}\left(\mathbf{B}_{-i}\right), \sum_{j} \hat{a}_{i j} \leq c_{i}$.

Proof In order to find $a_{i}\left(B_{-i}\right)$ we define the truncated bid vector $\tilde{\mathbf{b}}_{-i}$ as follows. For any set $S \subseteq[m]$ of resources, we denote by $\mathbf{1}_{S}$ the indicator vector w.r.t. $S$, such that $x_{j}=1$ for $j \in S$ and $x_{j}=0$ otherwise. For any vector $p_{i}$ and any $\lambda>0$, let $T:=T\left(\lambda, p_{i}\right)$ be a maximal subset of resources such that, $v_{i}^{c_{i}}\left(\mathbf{1}_{T}\right)<$ $\frac{1}{\lambda} \sum_{j \in T} o_{i j} p_{i j}$. For every $k \neq i$, if $j \in T$, then $\tilde{b}_{k j}=0$, otherwise $\tilde{b}_{k j}=b_{k j}$. Similarly with $p_{i}$, we define the vector $\tilde{p}_{i}=\left(\tilde{p}_{i j}\right)_{j}$, where $\tilde{p}_{i j}=\sum_{k \neq i} \tilde{b}_{k j}$ for all $j \in[m]$. Moreover, if $\mathbf{b}_{-i} \sim \mathbf{B}_{-i}$, then $p_{i}$ is an induced random variable with distribution denoted by $P_{i}=\left\{p_{i} \mid \mathbf{b}_{-i} \sim \mathbf{B}_{-i}\right\}$. We further define distributions $\tilde{\mathbf{B}}_{-i}$ and $\tilde{P}_{i}$, as $\tilde{\mathbf{B}}_{-i}=\left\{\tilde{\mathbf{b}}_{-i} \mid \mathbf{b}_{-i} \sim \mathbf{B}_{-i}\right\}$ and $\tilde{P}_{i}=\left\{\tilde{p}_{i} \mid \tilde{\mathbf{b}}_{-i} \sim \tilde{\mathbf{B}}_{-i}\right\}$.

Now consider the following bidding strategy $a_{i}\left(\mathbf{B}_{-i}\right)$ : sampling $b_{i}^{\prime} \sim \tilde{P}_{i}$ and bid$\operatorname{ding} a_{i j}=\frac{1}{\lambda} o_{i j} b_{i j}^{\prime}$ for each resource $j$. We first show $\sum_{j \in[m]} a_{i j} \leq c_{i}$. It is sufficient to show that $\sum_{j \notin T} a_{i j} \leq v_{i}^{c_{i}}\left(\mathbf{1}_{[m] \backslash T}\right)$ since $v_{i}^{c_{i}}\left(\mathbf{1}_{[m] \backslash T}\right) \leq c_{i}$ and $\sum_{j \in T} a_{i j}=0$. For the sake of contradiction suppose $v_{i}^{c_{i}}\left(\mathbf{1}_{[m] \backslash T}\right)<\sum_{j \notin T} a_{i j}$. Then, by the definition of $T$ and $\tilde{p}_{i}$ and due to subadditivity $v_{i}^{c_{i}}\left(\mathbf{1}_{[m]}\right) \leq v_{i}^{c_{i}}\left(\mathbf{1}_{T}\right)+v_{i}^{c_{i}}\left(\mathbf{1}_{[m] \backslash T}\right)<$ $\frac{1}{\lambda} \sum_{j \in T} o_{i j} p_{i j}+\sum_{j \notin T} a_{i j}=\frac{1}{\lambda} \sum_{j \in[m]} o_{i j} p_{i j}$, which contradicts the maximality of $T$.

Next we show that, for any bid $b_{i}$ and $\lambda>0$,

$$
v_{i}^{c_{i}}\left(x_{i}\left(b_{i}, \mathbf{B}_{-i}\right)\right)+\frac{1}{\lambda} \sum_{j \in[m]} o_{i j} \underset{\tilde{p}_{i} \sim \tilde{P}_{i}}{\mathbb{E}}\left[p_{i j}\right] \geq v_{i}^{c_{i}}\left(x_{i}\left(b_{i}, \tilde{\mathbf{B}}_{-i}\right)\right)+\frac{1}{\lambda} \sum_{j \in[m]} o_{i j} \underset{\tilde{p}_{i} \sim \tilde{P}_{i}}{\mathbb{E}}\left[\tilde{p}_{i j}\right] .
$$

Observe that $x_{i}\left(b_{i}, \tilde{\mathbf{b}}_{-i}\right) \leq x_{i}\left(b_{i}, \mathbf{b}_{-i}\right)+\mathbf{1}_{T}$. Therefore, by using the subadditivity of $v_{i}^{c_{i}}$ and the definitions of $T$ and $\tilde{p}_{i}$

$$
\begin{aligned}
v_{i}^{c_{i}}\left(x_{i}\left(b_{i}, \tilde{\mathbf{b}}_{-i}\right)\right) & \leq v_{i}^{c_{i}}\left(x_{i}\left(b_{i}, \mathbf{b}_{-i}\right)\right)+v_{i}^{c_{i}}\left(\mathbf{1}_{T}\right) \leq v_{i}^{c_{i}}\left(x_{i}\left(b_{i}, \mathbf{b}_{-i}\right)\right)+\frac{1}{\lambda} \sum_{j \in T} o_{i j} p_{i j} \\
& =v_{i}^{c_{i}}\left(x_{i}\left(b_{i}, \mathbf{b}_{-i}\right)\right)+\frac{1}{\lambda} \sum_{j \in[m]} o_{i j} p_{i j}-\frac{1}{\lambda} \sum_{j \in[m]} o_{i j} \tilde{p}_{i j} .
\end{aligned}
$$


The claim follows by rearranging terms and taking the expectation of $\mathbf{b}_{-i}, \tilde{\mathbf{b}}_{-i}, p_{i}$ and $\tilde{p}_{i}$ over $\mathbf{B}_{-i}, \tilde{\mathbf{B}}_{-i}, P_{i}$ and $\tilde{P}_{i}$, respectively. We next prove the statement of the lemma.

$$
\begin{aligned}
& \mathbb{E}_{b_{i}^{\prime} \sim \tilde{P}_{i}}\left[u_{i}\left(\frac{1}{\lambda} o_{i} b_{i}^{\prime}, \mathbf{B}_{-i}\right)\right]=\underset{b_{i}^{\prime} \sim \tilde{P}_{i}}{\mathbb{E}}\left[v_{i}\left(\frac{1}{\lambda} o_{i} b_{i}^{\prime}, \mathbf{B}_{-i}\right)\right]-\frac{1}{\lambda} \sum_{j \in[m]} o_{i j} \underset{b_{i}^{\prime} \sim \tilde{P}_{i}}{\mathbb{E}}\left[b_{i j}^{\prime}\right] \\
& \geq \underset{b_{i}^{\prime} \sim \tilde{P}_{i}}{\mathbb{E}}\left[v_{i}^{c_{i}}\left(\frac{1}{\lambda} o_{i} b_{i}^{\prime}, \mathbf{B}_{-i}\right)\right]-\frac{1}{\lambda} \sum_{j \in[m]} o_{i j} \underset{\tilde{p}_{i} \sim \tilde{P}_{i}}{\mathbb{E}}\left[\tilde{p}_{i j}\right] \quad \text { (by definition of } v_{i}^{c_{i}} \text { ) } \\
& \geq \underset{b_{i}^{\prime} \sim \tilde{P}_{i}}{\mathbb{E}}\left[v_{i}^{c_{i}}\left(\frac{1}{\lambda} o_{i} b_{i}^{\prime}, \tilde{\mathbf{B}}_{-i}\right)\right]-\frac{1}{\lambda} \sum_{j \in[m]} o_{i j} \underset{\tilde{p}_{i} \sim \tilde{P}_{i}}{\mathbb{E}}\left[p_{i j}\right] \quad \text { (by Inequality (1)) } \\
& \geq \frac{1}{2} \underset{b_{i}^{\prime} \sim \tilde{P}_{i} \tilde{p}_{i} \sim \tilde{P}_{i}}{\mathbb{E}}\left[v_{i}^{c_{i}}\left(\frac{o_{i} b_{i}^{\prime}}{o_{i} b_{i}^{\prime}+\lambda \tilde{p}_{i}}+\frac{o_{i} \tilde{p}_{i}}{o_{i} \tilde{p}_{i}+\lambda b_{i}^{\prime}}\right)\right]-\frac{1}{\lambda} \sum_{j \in[m]} o_{i j} \sum_{k \neq i} \underset{b_{k j} \sim B_{k j}}{\mathbb{E}}\left[b_{k j}\right] \\
& \text { (by swapping } b_{i}^{\prime} \text { with } \tilde{p}_{i} \text { and the subadditivity of } v_{i}^{c_{i}}(\cdot) \text { ) } \\
& \left.\geq \quad \frac{1}{2} \underset{b_{i}^{\prime} \sim \tilde{P}_{i} \tilde{p}_{i} \sim \tilde{P}_{i}}{\mathbb{E}} \underset{v_{i}}{\mathbb{E}}\left[v_{i}^{c_{i}}\left(\frac{b_{i}^{\prime}}{b_{i}^{\prime}+\lambda \tilde{p}_{i}}+\frac{\tilde{p}_{i}}{\tilde{p}_{i}+\lambda b_{i}^{\prime}}\right)\right)\right]-\frac{1}{\lambda} \sum_{j \in[m]} o_{i j} \sum_{k \in[n]} \mathbb{E}\left[b_{k j}\right] \\
& \geq \frac{1}{2} v_{i}^{c_{i}}\left(\frac{2 o_{i}}{\lambda+1}\right)-\frac{1}{\lambda} \sum_{j \in[m]} \sum_{k \in[n]} o_{i j} \mathbb{E}\left[b_{k j}\right] \quad \text { (by monotonicity of } v_{i}^{c_{i}} \text { ) } \\
& \geq \frac{1}{\lambda+1} v_{i}^{c_{i}}\left(o_{i}\right)-\frac{1}{\lambda} \sum_{j \in[m]} \sum_{k \in[n]} o_{i j} \mathbb{E}\left[b_{k j}\right] .\left(\text { subadditivity of } v_{i}^{c_{i}} ; \frac{2}{\lambda+1} \leq 1\right)
\end{aligned}
$$

For the second inequality, notice that the second term doesn't depend on $b_{i}^{\prime}$ and so, we apply Inequality (1) for every $b_{i}^{\prime}$. For the forth inequality we used the fact that $o_{i} \leq 1$ in the first term and the inequality $\sum_{k \neq i} \mathbb{E}\left[b_{k j}\right] \leq \sum_{k \in[n]} \mathbb{E}\left[b_{k j}\right]$ in the second term. For the fifth inequality, it is not hard to see that, for every $b_{i}^{\prime}, \tilde{p}_{i}$ and $\lambda \geq 1$, it holds that $\frac{b_{i}^{\prime}}{b_{i}^{\prime}+\lambda \tilde{p}_{i}}+\frac{\tilde{p}_{i}}{\tilde{p}_{i}+\lambda b_{i}^{\prime}} \geq \frac{2}{\lambda+1}$.

We are ready to show the PoA bound by using the above lemma.

Theorem 15 The coarse correlated PoA for the proportional allocation mechanism when agents have budget constraints and subadditive valuations, is at most $\phi+1 \approx$ 2.618 .

Proof Suppose B is a coarse correlated equilibrium. Let $A$ be the set of agents such that for every $i \in A, v_{i}(\mathbf{B}) \leq c_{i}$. For simplicity, we use $v_{i}^{c_{i}}(\mathbf{B})$ to denote $\min \left\{\mathbb{E}_{\mathbf{b} \sim \mathbf{B}}\left[v_{i}\left(x_{i}(\mathbf{b})\right)\right], c_{i}\right\}$. Then for all $i \notin A, v_{i}^{c_{i}}(\mathbf{B})=c_{i} \geq v_{i}^{c_{i}}\left(o_{i}\right)$ and $v_{i}^{c_{i}}(\mathbf{B})=$ $c_{i} \geq \sum_{j \in[m]} \mathbb{E}\left[b_{i j}\right]$. The latter inequality comes from that agents do not bid higher than their budgets. Let $\lambda=\phi$. So $1-1 / \lambda=1 /(1+\lambda)$. By taking the linear combination and summing up over all agents not in $A$ we get

$$
\sum_{i \notin A} v_{i}^{c_{i}}(\mathbf{B}) \geq \frac{1}{\lambda+1} \sum_{i \notin A} v_{i}^{c_{i}}\left(o_{i}\right)+\frac{1}{\lambda} \sum_{i \notin A} \sum_{j \in[m]} \mathbb{E}\left[b_{i j}\right] .
$$


For every $i \in A$, we consider the deviating bidding strategy $a_{i}\left(\mathbf{B}_{-i}\right)$ that is described in Lemma 14, then

$$
\begin{aligned}
v_{i}^{c_{i}}(\mathbf{B}) & =v_{i}\left(x_{i}(\mathbf{B})\right)=u_{i}\left(x_{i}(\mathbf{B})\right)+\sum_{j \in[m]} \mathbb{E}\left[b_{i j}\right] \geq u_{i}\left(a_{i}\left(\mathbf{B}_{-i}\right), \mathbf{B}_{-i}\right)+\frac{1}{\lambda} \sum_{j \in[m]} \mathbb{E}\left[b_{i j}\right] \\
& \geq \frac{1}{\lambda+1} v_{i}^{c_{i}}\left(o_{i}\right)-\frac{1}{\lambda} \sum_{j \in[m]} \sum_{k \in[n]} o_{i j} \mathbb{E}\left[b_{k j}\right]+\frac{1}{\lambda} \sum_{j \in[m]} \mathbb{E}\left[b_{i j}\right] .
\end{aligned}
$$

By summing up over all $i \in A$ and by combining with inequality (2) we get

$$
\begin{aligned}
& \sum_{i \in[n]} \min \left\{v_{i}\left(x_{i}(\mathbf{B})\right), c_{i}\right\} \\
\geq & \frac{1}{\lambda+1} \sum_{i \in[n]} v_{i}^{c_{i}}\left(o_{i}\right)+\frac{1}{\lambda} \sum_{i \in[n]} \sum_{j \in[m]} \mathbb{E}\left[b_{i j}\right]-\frac{1}{\lambda} \sum_{i \in A} \sum_{j \in[m]} \sum_{k \in[n]} o_{i j} \mathbb{E}\left[b_{k j}\right] \\
\geq & \frac{1}{\lambda+1} \sum_{i \in[n]} v_{i}^{c_{i}}\left(o_{i}\right) . \quad\left(\text { since } \sum_{i \in A} o_{i j} \leq 1\right)
\end{aligned}
$$

Therefore, the PoA with respect to the effective welfare is at most $\phi+1$. (recall that for Inequality (2) we set $\lambda=\phi$ )

In the Bayesian case we cannot apply the same ideas for subadditive valuations due to the fact that in the definition of $\mathbb{E}[\mathrm{EW}]$ the expectation over $v_{-i}$ appears inside the minimum function (see Section 2). However, by applying Jensen's inequality for concave functions our upper bound also holds for the Bayesian case with singleresource and concave valuations. Notice that the lower bound of Theorem 4 also holds under the restriction of budgets and therefore, we couldn't expect the same upper bound for concave valuations and many resources.

Theorem 16 The Bayesian PoA of single-resource proportional allocation games is at most $\phi+1 \approx 2.618$, when agents have budget constraints and concave valuations.

Proof Suppose B is a Bayesian Nash equilibrium. Recall that in the Bayesian setting, agent $i$ 's type $t_{i}=\left(v_{i}, c_{i}\right)$ are drawn from some know distribution independently. We use the notation $\mathbf{C}=\left(C_{1}, C_{2}, \ldots, C_{n}\right)$ to denote the bidding distribution in $\mathbf{B}$ which includes the randomness of bidding strategy $\mathbf{b}$ and agents' types $\mathbf{t}$, that is, $b_{i}\left(t_{i}\right) \sim C_{i}$. Then the utility of agent $i$ with type $t_{i}$ is $u_{i}\left(B_{i}\left(t_{i}\right), \mathbf{C}_{-i}\right)$. Notice that $\mathbf{C}_{-i}$ does not depend on any particular $\mathbf{t}_{-i}$.

Recall that $v^{c}(x)=\min \{v(x), c\}$. It is easy to check $v^{c}$ is concave if $v$ is concave. For any agents types $\mathbf{t}=(\mathbf{v}, \mathbf{c})$, let $\mathbf{o}^{\mathbf{t}}=\left(o_{1}^{\mathbf{t}}, \ldots, o_{n}^{\mathbf{t}}\right)$ be the allocation vector that maximizes the effective welfare. We define $o_{i}^{t_{i}}$ to be the expected allocation over $\mathbf{t}_{-i} \sim \mathbf{D}_{-i}$ to agent $i$ in the optimum solution with respect to effective welfare when her type is $t_{i}$. Formally, $o_{i}^{t_{i}}=\mathbb{E}_{\mathbf{t}_{-i} \sim \mathbf{D}_{-i}}\left[o_{i}^{\left(t_{i}, \mathbf{t}_{-i}\right)}\right]$.

For every agent $i$, let $A_{i}$ be the set of $t_{i}$ such that $v_{i}\left(x_{i}\left(B_{i}\left(t_{i}\right), \mathbf{C}_{-i}\right)\right) \leq c_{i}$. For simplicity, we use $v_{i}^{c_{i}}\left(B_{i}\left(t_{i}\right), \mathbf{C}_{-i}\right)$ to denote $\min \left\{\mathbb{E}_{\mathbf{t}_{-i}, \mathbf{b} \sim \mathbf{B}(\mathbf{t})}\left[v_{i}\left(x_{i}(\mathbf{b})\right)\right], c_{i}\right\}$. For every 
$t_{i} \notin A_{i}, v_{i}^{c_{i}}\left(B_{i}\left(t_{i}\right), \mathbf{C}_{-i}\right)=c_{i} \geq \min \left\{\mathbb{E}_{\mathbf{t}_{-i}}\left[v_{i}\left(o_{i}^{\mathbf{t}}\right)\right], c_{i}\right\}$ and $v_{i}^{c_{i}}\left(B_{i}\left(t_{i}\right), \mathbf{C}_{-i}\right)=c_{i} \geq$ $\mathbb{E}\left[b_{i}\left(t_{i}\right)\right]$. The latter inequality comes from the fact that agents do not bid above their budget. Let $\lambda=\phi$. So $1-1 / \lambda=1 /(1+\lambda)$. By taking the linear combination, taking the expectation over all $t_{i} \notin A_{i}$ and summing up over all agents we get

$$
\sum_{i \in[n]} \underset{t_{i} \notin A_{i}}{\mathbb{E}}\left[v_{i}^{c_{i}}\left(B_{i}\left(t_{i}\right), \mathbf{C}_{-i}\right)\right] \geq \sum_{i \in[n]} \underset{t_{i} \notin A_{i}}{\mathbb{E}}\left[\frac{1}{\lambda+1} \min \left\{\underset{\mathbf{t}_{-i}}{\mathbb{E}}\left[v_{i}\left(o_{i}^{\mathbf{t}}\right)\right], c_{i}\right\}+\frac{1}{\lambda} \mathbb{E}\left[b_{i}\left(t_{i}\right)\right]\right]
$$

For every $t_{i} \in A_{i}$, by Lemma 14, there exists a randomized bid $a_{i}\left(t_{i}, \mathbf{B}_{-i}\right)$ for agent $i$, such that, for any $\lambda \geq 1: u_{i}\left(a_{i}\left(t_{i}, \mathbf{B}_{-i}\right), \mathbf{B}_{-i}\right) \geq \frac{1}{\lambda+1} v_{i}^{c_{i}}\left(o_{i}^{t_{i}}\right)-\frac{1}{\lambda} o_{i}^{t_{i}} \sum_{k \neq i} \mathbb{E}\left[b_{k}\right]$. By the definition of equilibria

$$
\begin{aligned}
& v_{i}^{c_{i}}\left(B_{i}\left(t_{i}\right), \mathbf{C}_{-i}\right)=v_{i}\left(B_{i}\left(t_{i}\right), \mathbf{C}_{-i}\right)=u_{i}\left(B_{i}\left(t_{i}\right), \mathbf{C}_{-i}\right)+\mathbb{E}\left[b_{i}\left(t_{i}\right)\right] \\
\geq & u_{i}\left(a_{i}\left(t_{i}, \mathbf{C}_{-i}\right), \mathbf{C}_{-i}\right)+\frac{1}{\lambda} \mathbb{E}\left[b_{i}\left(t_{i}\right)\right] \geq \frac{1}{\lambda+1} v_{i}^{c_{i}}\left(o_{i}^{t_{i}}\right)-\frac{1}{\lambda} o_{i}^{t_{i}} \sum_{k \in[n]} \mathbb{E}\left[b_{k}\right]+\frac{1}{\lambda} \mathbb{E}\left[b_{i}\left(t_{i}\right)\right] \\
\geq & \frac{1}{\lambda+1} \min \left\{\underset{\mathbf{t}_{-i}}{\mathbb{E}}\left[v_{i}\left(o_{i}^{\mathbf{t}}\right)\right], c_{i}\right\}-\frac{1}{\lambda} o_{i}^{t_{i}} \sum_{k \in[n]} \mathbb{E}\left[b_{k}\right]+\frac{1}{\lambda} \mathbb{E}\left[b_{i}\left(t_{i}\right)\right] .
\end{aligned}
$$

The last inequality holds due to Jensen's inequality for concave functions. By taking the expectation over all $t_{i} \in A_{i}$, summing over all agents and combining with inequality (3):

$$
\begin{aligned}
& \sum_{i} \underset{t_{i}}{\mathbb{E}}\left[v_{i}^{c_{i}}\left(B_{i}\left(t_{i}\right), \mathbf{C}_{-i}\right)\right] \\
\geq & \frac{1}{\lambda+1} \sum_{i} \underset{t_{i}}{\mathbb{E}}\left[\min \left\{\underset{\mathbf{t}_{-i}}{\mathbb{E}}\left[v_{i}\left(o_{i}^{\mathbf{t}}\right)\right], c_{i}\right\}\right]+\frac{1}{\lambda} \sum_{i} \mathbb{E}\left[b_{i}\right]-\frac{1}{\lambda} \sum_{i} \underset{t_{i} \in A_{i}}{\mathbb{E}}\left[o_{i}^{t_{i}}\right] \sum_{k \in[n]} \mathbb{E}\left[b_{k}\right] \\
\geq & \frac{1}{\lambda+1} \sum_{i} \underset{t_{i}}{\mathbb{E}}\left[\min \left\{\underset{\mathbf{t}_{-i}}{\mathbb{E}}\left[v_{i}\left(o_{i}^{\mathbf{t}}\right)\right], c_{i}\right\}\right]+\frac{1}{\lambda} \sum_{i} \mathbb{E}\left[b_{i}\right]-\frac{1}{\lambda} \sum_{k \in[n]} \mathbb{E}\left[b_{k}\right] \\
= & \frac{1}{\lambda+1} \sum_{i} \underset{t_{i}}{\mathbb{E}}\left[\min \left\{\underset{\mathbf{t}_{-i}}{\mathbb{E}}\left[v_{i}\left(o_{i}^{\mathbf{t}}\right)\right], c_{i}\right\}\right] .
\end{aligned}
$$

The first inequality is due to that $\sum_{i} \mathbb{E}_{t_{i}}\left[o_{i}^{t_{i}}\right]=\sum_{i} \mathbb{E}_{\mathbf{t}}\left[o_{i}^{\mathbf{t}}\right]=\mathbb{E}_{\mathbf{t}}\left[\sum_{i} o_{i}^{\mathbf{t}}\right] \leq 1$, since for every $\mathbf{t}, \sum_{i} o_{i}^{\mathbf{t}} \leq 1$. Therefore, the PoA is at most $\phi+1$.

Remark 17 Syrgkanis and Tardos [26] compared the social welfare in the equilibrium with the effective welfare in the optimum allocation. Caragiannis and Voudouris [3] also give an upper bound of 2 for this ratio in the single resource case. We can obtain 
the same upper bound by replacing $\lambda$ with 1 in Lemma 14 and following the ideas of Theorems 7 and 8 .

\section{Polyhedral Environment}

In this section, we study the efficiency of the proportional allocation mechanism in the polyhedral environment, that was previously studied by Nguyen and Tardos [20]. We show a tight price of anarchy bound of 2 for agents with subadditive valuations. Recall that, in this setting, the allocation to each agent $i$ is now represented by a single parameter $x_{i}$, and not by a vector $\left(x_{i 1}, \ldots, x_{i m}\right)$. In addition, any feasible allocation vector $\mathbf{x}=\left(x_{1}, \ldots, x_{n}\right)$ should satisfy a polyhedral constraint $A \cdot \mathbf{x} \leq \mathbf{1}$, where $A$ is a non-negative $m \times n$ matrix and each row of $A$ corresponds to a different resource and $\mathbf{1}$ is a vector with all ones. Each agent aims to maximize her utility $u_{i}=$ $v_{i}\left(x_{i}\right)-q_{i}$, where $v_{i}$ is a subadditive function representing the agent's valuation. The proportional allocation mechanism determines the following allocation and payments for each agent:

$$
x_{i}(\mathbf{b})=\min _{j: a_{i j}>0}\left\{\frac{b_{i j}}{a_{i j} \sum_{k \in[n]} b_{k j}}\right\} ; \quad q_{i}(\mathbf{b})=\sum_{j \in[m]} b_{i j},
$$

where $a_{i j}$ is the $(i, j)$-th entry of matrix $A$. It is easy to verify that the above allocation satisfies the polyhedral constraints.

Theorem 18 If agents have subadditive valuations, the pure PoA of the proportional allocation mechanism in the polyhedral environment is exactly 2.

Proof We first show that the PoA is at most 2. Let $\mathbf{o}=\left\{o_{1}, \ldots, o_{n}\right\}$ be the optimal allocation, $\mathbf{b}$ be a pure Nash Equilibrium, and let $p_{i j}=\sum_{k \neq i} b_{i j}$. For each agent $i$, consider the deviating bid $b_{i}^{\prime}$ such that $b_{i j}^{\prime}=o_{i} a_{i j} p_{i j}$ for all resources $j$. Since $\mathbf{b}$ is a Nash Equilibrium,

$$
\begin{aligned}
u_{i}(\mathbf{b}) & \geq u_{i}\left(b_{i}^{\prime}, \mathbf{b}_{-i}\right)=v_{i}\left(\min _{j: a_{i j}>0}\left\{\frac{o_{i} a_{i j} p_{i j}}{a_{i j}\left(p_{i j}+o_{i} a_{i j} p_{i j}\right)}\right\}\right)-\sum_{j \in[m]} o_{i} a_{i j} p_{i j} \\
& \geq v_{i}\left(\frac{o_{i}}{2}\right)-\sum_{j \in[m]} o_{i} a_{i j} p_{i j} \geq \frac{1}{2} v_{i}\left(o_{i}\right)-\sum_{j \in[m]} o_{i} a_{i j} p_{i j} .
\end{aligned}
$$

The second inequality is true since $A \cdot \mathbf{x} \leq \mathbf{1}$, for every allocation $\mathbf{x}$, and therefore $o_{i} a_{i j}<1$. The last inequality holds due to subadditivity of $v_{i}$. By summing up over all agents we get

$$
\sum_{i} u_{i}(\mathbf{b}) \geq \frac{1}{2} \sum_{i} v_{i}\left(o_{i}\right)-\sum_{j \in[m]} \sum_{i \in[n]} o_{i} a_{i j} p_{i j} \geq \frac{1}{2} \sum_{i} v_{i}\left(o_{i}\right)-\sum_{j \in[m]} \sum_{k \in[n]} b_{k j} .
$$


The last inequality holds due to the fact that $p_{i j} \leq \sum_{k \in[n]} b_{k j}$ and $\sum_{i \in[n]} o_{i} a_{i j} \leq 1$. The fact that $\mathrm{PoA} \leq 2$ follows by rearranging the terms.

For the lower bound, consider the lower bound instance of Theorem 9, where the polyhedral constraint is given by $x_{1}+x_{2} \leq 1$.

Open Access This article is distributed under the terms of the Creative Commons Attribution 4.0 International License (http://creativecommons.org/licenses/by/4.0/), which permits unrestricted use, distribution, and reproduction in any medium, provided you give appropriate credit to the original author(s) and the source, provide a link to the Creative Commons license, and indicate if changes were made.

\section{References}

1. Anshelevich, E., Dasgupta, A., Kleinberg, J.M., Tardos, É., Wexler, T., Roughgarden, T.: The price of stability for network design with fair cost allocation. SIAM J. Comput. 38(4), 1602-1623 (2008)

2. Bhawalkar, K., Roughgarden, T.: Welfare guarantees for combinatorial auctions with item bidding. In: Proceedings of the Twenty-Second Annual ACM-SIAM Symposium on Discrete Algorithms, SODA 2011, pp. 700-709, California, USA (2011)

3. Caragiannis, I., Voudouris, A.A.: Welfare guarantees for proportional allocations. In: Algorithmic Game Theory - 7th International Symposium, SAGT 2014, pp. 206-217. Proceedings, Haifa, Israel (2014)

4. Chen, H.-L., Roughgarden, T., Valiant, G.: Designing network protocols for good equilibria. SIAM J. Comput. 39(5), 1799-1832 (2010)

5. Christodoulou, G., Kovács, A., Schapira, M.: Bayesian combinatorial auctions. In: Automata, Languages and Programming, 35th International Colloquium, ICALP 2008, pp. 820-832. Proceedings, Part I: Tack A: Algorithms, Automata, Complexity, and Games, Reykjavik, Iceland (2008)

6. Christodoulou, G., Kovács, A., Sgouritsa, A., Tang, B.: Tight bounds for the price of anarchy of simultaneous first-price auctions. ACM Trans. Econ. Comput. 4(2), 9 (2016)

7. Christodoulou, G., Sgouritsa, A., Tang, B.: On the efficiency of the proportional allocation mechanism for divisible resources. In: Algorithmic Game Theory - 8th International Symposium, SAGT 2015, pp. 165-177. Proceedings, Saarbrücken, Germany (2015)

8. Chun, B.N., Culler, D.E.: Market-based proportional resource sharing for clusters. Technical Report, University of California at Berkeley, CA, USA (2000)

9. Correa, J.R., Schulz, A.S., Stier-Moses, N.E.: The price of anarchy of the proportional allocation mechanism revisited. In: Web and Internet Economics - 9th International Conference, WINE 2013, pp. 109-120. Proceedings, MA, USA (2013)

10. Dobzinski, S., Paes Leme, R.: Efficiency guarantees in auctions with budgets. In: Automata, Languages, and Programming - 41st International Colloquium, ICALP 2014, pp. 392-404. Proceedings, Part I, Copenhagen, Denmark (2014)

11. Dobzinski, S., Nisan, N., Schapira, M.: Approximation algorithms for combinatorial auctions with complement-free bidders. Math. Oper Res. 35(1), 1-13 (2010)

12. Evans, D.S., Heckman, J.J.: A test for subadditivity of the cost function with an application to the bell system. Amer. Econ. Rev., 615-623 (1984)

13. Feldman, M., Fu, H., Gravin, N., Lucier, B.: Simultaneous auctions are (almost) efficient, CA, USA (2013)

14. Feldman, M., Lai, K., Zhang, L.: A price-anticipating resource allocation mechanism for distributed shared clusters. In: Proceedings 6th ACM Conference on Electronic Commerce (EC-2005), pp. 127136, BC, Canada (2005)

15. Hassidim, A., Kaplan, H., Mansour, Y., Nisan, N.: Non-price equilibria in markets of discrete goods. In: Proceedings 12th ACM Conference on Electronic Commerce (EC-2011), pp. 295-296, CA, USA (2011)

16. Johari, R., Tsitsiklis, J.N.: Efficiency loss in a network resource allocation game. Math. Oper Res. 29(3), 407-435 (2004) 
17. Johari, R., Tsitsiklis, J.N.: Efficiency of scalar-parameterized mechanisms. Oper. Res. 57(4), 823-839 (2009)

18. Kelly, F.: Charging and rate control for elastic traffic. Eur. Trans. Telecommun. 8(1), 33-37 (1997)

19. Koutsoupias, E., Papadimitriou, C.H.: Worst-case equilibria. In: STACS 99, 16th Annual Symposium on Theoretical Aspects of Computer Science, pp. 404-413. Proceedings, Trier, Germany (1999)

20. Nguyen, T., Tardos, É.: Approximately maximizing efficiency and revenue in polyhedral environments. In: Proceedings 8th ACM Conference on Electronic Commerce (EC-2007), pp. 11-19, California, USA (2007)

21. Nisan, N.: The communication complexity of approximate set packing and covering. In: Automata, Languages and Programming, 29th International Colloquium, ICALP 2002, pp. 868-875. Proceedings, Malaga, Spain (2002)

22. Rosenbaum, R.A.: Sub-additive functions. Duke Math. J. 17(3), 227-247, 09 (1950)

23. Roughgarden, T.: Barriers to near-optimal equilibria. In: 55th IEEE Annual Symposium on Foundations of Computer Science, FOCS 2014, pp. 71-80, PA, USA (2014)

24. Roughgarden, T.: The price of anarchy in games of incomplete information. ACM Trans. Econ. Comput. 3(1), 6 (2015)

25. Roughgarden, T., Tardos, É.: How bad is selfish routing? J. ACM 49(2), 236-259 (2002)

26. Syrgkanis, V., Tardos, É.: Composable and efficient mechanisms, CA, USA (2013)

27. Zhang, L.: The Efficiency and Fairness of a Fixed Budget Resource Allocation Game. In: Automata, Languages and Programming, 32nd International Colloquium, ICALP 2005, Lisbon, Portugal, July 11-15, 2005, Proceedings, pp. 485-496 (2005). doi:10.1007/11523468_40 